\title{
The sector accounts at a glance
}

\section{Net lending/borrowing}

Net borrowing by general government increased in 2009 to $£ 150.4$ billion, compared with net borrowing of $£ 68.6$ billion in 2008. The non-financial corporations sector was a net lender, with $£ 66.6$ billion in 2009 and $£ 35.1$ billion in 2008 . The households and non-profit institutions serving households (NPISH) sector was a net lender of $\mathrm{f27.3}$ billion in 2009, compared with a net borrower of $\mathrm{f} 41.0$ billion in 2008 . Financial corporations were net lenders in 2009 at $£ 43.9$ billion, from net lending of $£ 54.0$ billion in 2008 . Rest of the world sector net lending was $\mathrm{f} 12.3$ billion in 2009 , compared with f20.5 billion in 2008 .

\section{Net financial transactions}

Net financial transactions by general government showed a deficit of $f 151.2$ billion in 2009 , compared with a $£ 69.0$ billion deficit in 2008. Non-financial corporations show a surplus of $£ 58.0$ billion in 2009, compared with a $£ 44.2$ billion surplus in 2008. Households and NPISH showed a surplus of $£ 35.6$ billion in 2009 , compared with a deficit of $\mathrm{f} 49.8$ billion in 2008 . In 2009 financial corporations showed a surplus of $f 52.1$ billion, following a surplus of $£ 48.8$ billion in 2008 . The rest of the world sector showed a surplus of $\mathrm{f5.6}$ billion in 2009, compared with $\mathrm{f} 25.8$ billion in 2008 .
Net lending/borrowing, 2009

f billion

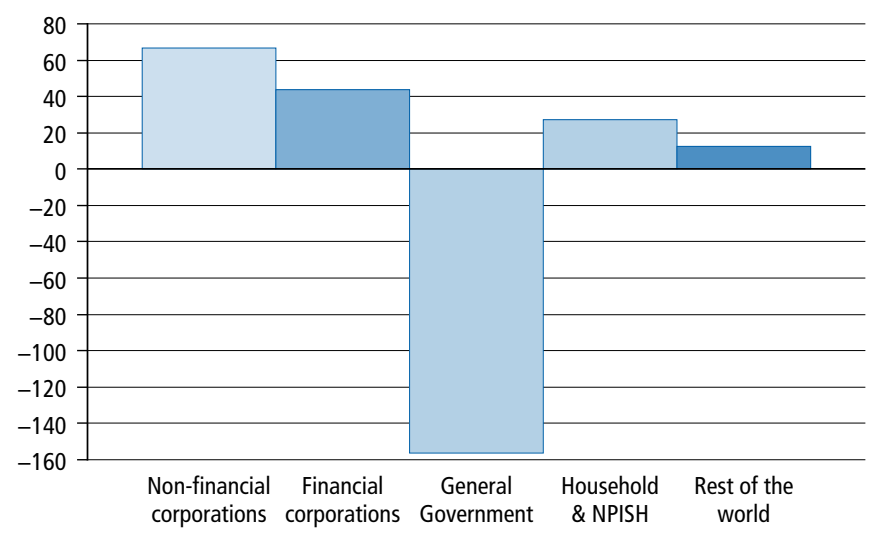

\section{Net financial transactions}

\section{f billion}

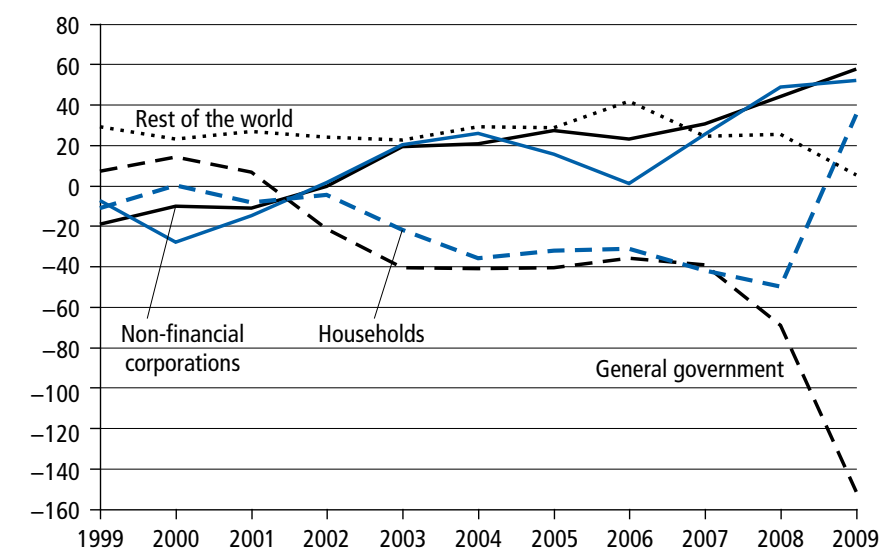




\section{Gross trading profits of private non-financial corporations}

Gross trading profit is the largest component of private non-financial corporations' gross operating surplus. Profits decreased by 10.2 per cent between 2008 and 2009 compared with an increase of 2.0 per cent between 2007 and 2008 .

\section{Real household disposable income}

Real household disposable income (RHDI) is the amount of money in real terms the household sector has available for spending, after taxes and other deductions. Between 2008 and 2009 RHDI increased by 1.8 per cent, compared with an increase of 1.1 per cent between 2007 and 2008.

\section{Household saving ratio}

The household saving ratio reflects household gross savings as a percentage of their total available resources (the amount available to invest or save). Household resources rose by 3.1 per cent between 2008 and 2009. Household and NPISH final consumption expenditure decreased by 2.1 per cent in the same period. As a consequence, the household saving ratio rose to 7.0 in 2009 from 2.0 in 2008.
Gross trading profits of private non-financial corporations

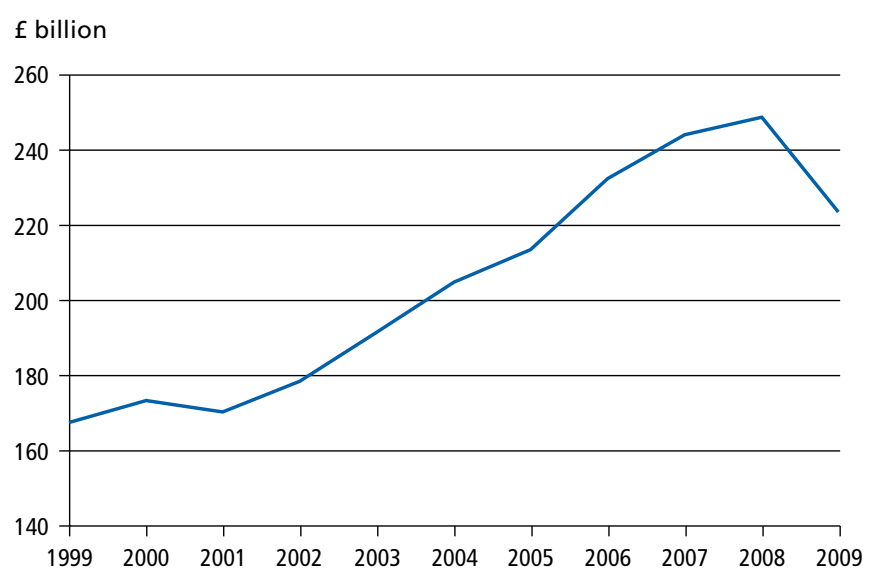

Annual changes in real household disposable income Per cent

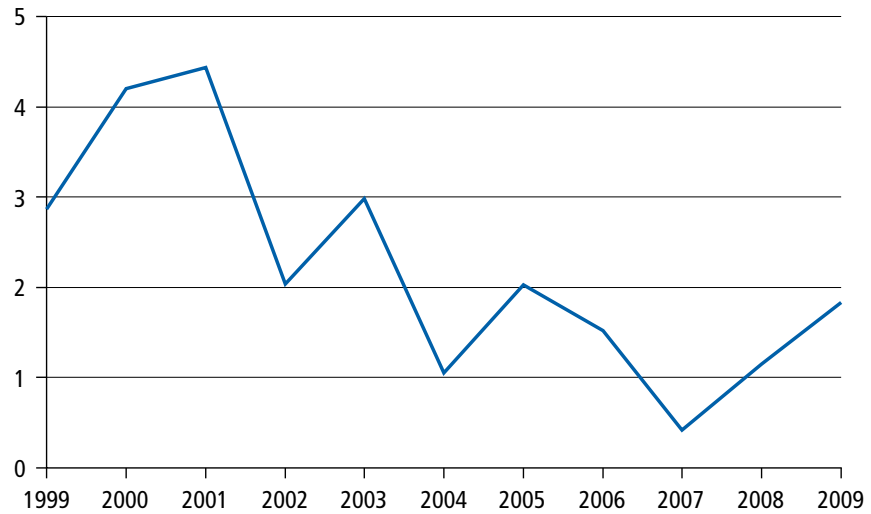

Household saving ratio

Per cent

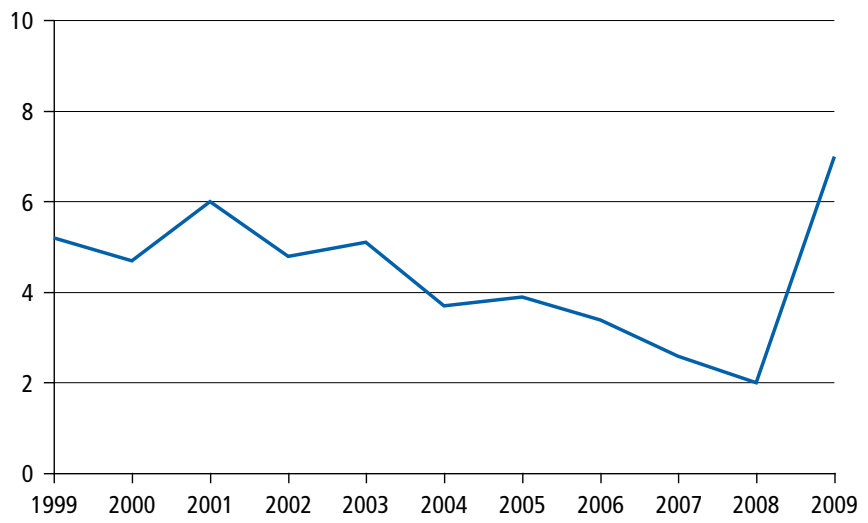


The sector accounts: Key economic indicators

$f$ million

\begin{tabular}{|c|c|c|c|c|c|c|c|c|c|c|c|}
\hline & & & 2001 & 2002 & 2003 & 2004 & 2005 & 2006 & 2007 & 2008 & 2009 \\
\hline \multicolumn{12}{|c|}{ Net lending/borrowing by: } \\
\hline B.9 & Non-financial corporations & EABO & -6366 & 10549 & 22971 & 32464 & 27110 & 32390 & 31267 & 35071 & 66640 \\
\hline B.9 & Financial corporations & NHCQ & -22123 & 4386 & 13451 & 18702 & 9017 & -492 & 25128 & 53973 & 43903 \\
\hline B.9 & General government & NNBK & 7660 & -20183 & -41173 & -41889 & -40156 & -35346 & -38770 & -68577 & -150378 \\
\hline B.9 & Households and NPISH's & NSSZ & 1044 & -12477 & -12091 & -32130 & -27310 & -40511 & -51541 & -41002 & 27295 \\
\hline B.9 & Rest of the world & NHRB & 19784 & 17725 & 16841 & 22853 & 31338 & 43959 & 33916 & 20535 & 12287 \\
\hline \multicolumn{12}{|c|}{ Private non-financial corporations } \\
\hline \multicolumn{12}{|c|}{ Gross trading profits } \\
\hline & Continental shelf profits & CAGJ & 18961 & 18328 & 17882 & 19014 & 23313 & 27311 & 26080 & 31969 & 21037 \\
\hline & Others & CAGK & 151364 & 160068 & 173584 & 185842 & 190286 & 205157 & 218168 & 216998 & 202555 \\
\hline & Rental of buildings & FCBW & 12394 & 12904 & 13891 & 14796 & 15819 & 16659 & 17448 & 21748 & 17428 \\
\hline & less Holding gains of inventories & $-D L Q Z$ & 438 & -2856 & -4266 & -2906 & -4378 & -4094 & -6156 & -6292 & -4425 \\
\hline B. $2 g$ & Gross operating surplus & NRJK & 183157 & 188444 & 201091 & 216746 & 225040 & 245033 & 255540 & 264423 & 236595 \\
\hline \multirow[t]{5}{*}{ B.6g } & Household gross disposable income & QWND & 700094 & 725267 & 760916 & 782637 & 817554 & 853095 & 881466 & 919510 & 948521 \\
\hline & individual consumption expenditure index $(2006=100)^{1}$ & YBFS & 90.3 & 91.6 & 93.4 & 95.0 & 97.3 & 100.0 & 102.9 & 106.1 & 107.5 \\
\hline & Real household disposable income: & & & & & & & & & & \\
\hline & Chained volume measures (Reference year 2006) & RVGK & 775651 & 791488 & 815076 & 823672 & 840358 & 853095 & 856644 & 866487 & 882352 \\
\hline & Index $(2006=100)$ & OSXR & 90.9 & 92.8 & 95.6 & 96.6 & 98.5 & 100.0 & 100.4 & 101.6 & 103.4 \\
\hline \multirow[t]{3}{*}{ B. $8 \mathrm{~g}$} & Gross saving & NSSH & 43243 & 35665 & 40017 & 29959 & 33471 & 30420 & 24315 & 19326 & 68186 \\
\hline & Households total resources & NSSU & 834590 & 873867 & 926247 & 9578121 & 1008634 & 1055229 & 1103037 & 141887 & 1183889 \\
\hline & Saving ratio, per cent & RVGL & 6.0 & 4.8 & 5.1 & 3.7 & 3.9 & 3.4 & 2.6 & 2.0 & 7.0 \\
\hline
\end{tabular}

1 Rounded to one decimal place 